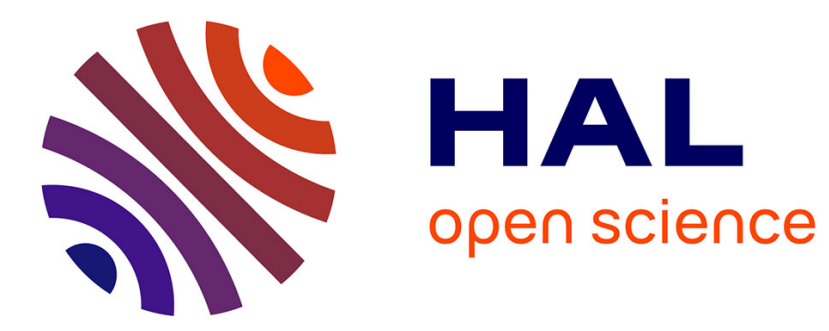

\title{
Consequences of a result on pure shear
}

$\mathrm{Ph}$. Boulanger, M. Hayes

\section{To cite this version:}

Ph. Boulanger, M. Hayes. Consequences of a result on pure shear. International Journal of Non-Linear Mechanics, 2007, 42 (2), pp.376. 10.1016/j.ijnonlinmec.2007.01.010 . hal-00501743

\section{HAL Id: hal-00501743 https://hal.science/hal-00501743}

Submitted on 12 Jul 2010

HAL is a multi-disciplinary open access archive for the deposit and dissemination of scientific research documents, whether they are published or not. The documents may come from teaching and research institutions in France or abroad, or from public or private research centers.
L'archive ouverte pluridisciplinaire HAL, est destinée au dépôt et à la diffusion de documents scientifiques de niveau recherche, publiés ou non, émanant des établissements d'enseignement et de recherche français ou étrangers, des laboratoires publics ou privés. 


\section{Author's Accepted Manuscript}

-

NON-LINEAR MECHANICS

Consequences of a result on pure shear

Ph. Boulanger, M. Hayes

PII:

S0020-7462(07)00050-9

DOI: doi:10.1016/j.ijnonlinmec.2007.01.010

Reference: NLM 1335

To appear in: International Journal of NonLinear Mechanics

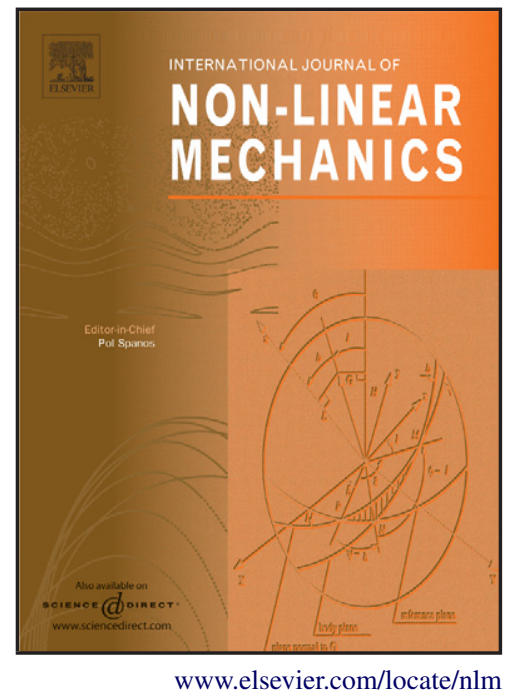

www.elsevier.com/locate/nlm

Received date: 3 November 2006

Revised date: 22 January 2007

Accepted date: 22 January 2007

Cite this article as: $\mathrm{Ph}$. Boulanger and $\mathrm{M}$. Hayes, Consequences of a result on pure shear, International Journal of Non-Linear Mechanics (2007), doi:10.1016/j.ijnonlinmec.2007.01.010

This is a PDF file of an unedited manuscript that has been accepted for publication. As a service to our customers we are providing this early version of the manuscript. The manuscript will undergo copyediting, typesetting, and review of the resulting galley proof before it is published in its final citable form. Please note that during the production process errors may be discovered which could affect the content, and all legal disclaimers that apply to the journal pertain. 


\title{
Consequences of a result on pure shear
}

\author{
Ph.BOULANGER \\ Département de Mathématique \\ Université Libre de Bruxelles \\ Campus Plaine C.P.218/1 \\ 1050 Bruxelles - Belgium

\section{M.HAYES} \\ School of Mathematical Sciences \\ University College Dublin \\ Belfield, Dublin 4 - Ireland \\ Dedicated to the memory of Ronald S. Rivlin, \\ father of modern continuum mechanics
}




\begin{abstract}
If, in a continuum, the Cauchy stress tensor is traceless, the material is said to be in a state of "pure shear". Here we derive consequences of a fundamental theorem concerning pure shear, in the contexts of infinitesimal strain, finite strain, and fluid motion.
\end{abstract}

\title{
1 Introduction
}

If the Cauchy stress tensor $\mathbf{t}$ at a point $P$ in a body is such that $\operatorname{tr} \mathbf{t}=0$, the material at $P$ is said to be in a state of "pure shear". It has been shown (see [1] and also [2] [3] [4] [5]) that then there exists an orthonormal triad $\mathbf{m}, \mathbf{n}, \mathbf{p}$ (say) such that $\mathbf{m} \cdot \mathbf{t m}=\mathbf{n} \cdot \mathbf{t n}=\mathbf{p} \cdot \mathbf{t p}=0$. In fact, there is an infinity of such triads. One of the elements of the triad may be arbitrarily chosen along any generator of a cone. Then, in [3], a simple geometrical determination of the other two is described.

Essentially, this is a result in tensor algebra (see [6], p.87, problem 10). Here, we exploit this result to obtain further consequences in the contexts of the linearized elasticity theory of isotropic bodies, the finite elasticity theory of isotropic bodies, and in the theory of viscous fluids.

The plan of the note is as follows. First $(\S 2)$ we recall basic notation and background results. Then $(\S 3)$ we consider infinitesimal strain of isotropic elastic bodies. Next $(\S 4)$ the finite strain theory of Bell and Ericksen models is considered ( $\$ 4)$. Finally $(\S 5)$ we close with a few remarks on fluid motion.

\section{Notation and background}

We consider real symmetric second order tensors : the Cauchy stress tensor $t_{i j}$, the infinitesimal strain tensor $e_{i j}$, the rate of strain tensor $d_{i j}$, and the left and right Cauchy-Green strain tensors $B_{i j}$ and $C_{A B}$, respectively.

We assume that a body is subjected to a deformation $\mathbf{x}=\mathbf{x}(\mathbf{X}, t)$ in which a particle initially at $\mathbf{X}$ is displaced to $\mathbf{X}$ at time $\mathbf{t}$ in the deformed state. We recall that the stretch $\lambda_{(\mathbf{N})}$ of an infinitesimal material line element initially along the unit vector $\mathbf{N}$ at $\mathbf{X}$ in the undeformed state of a body is given by $[7]$

$$
\lambda_{(\mathbf{N})}^{2}=\mathbf{N} \cdot \mathbf{C N}=N_{A} C_{A B} N_{B},
$$

and the "resile" $\lambda_{(\mathbf{n})}$ of an infinitesimal material line element along the unit vector $\mathbf{n}$ at $\mathbf{x}$ in the deformed state of the body is given by $[8]$

$$
\lambda_{(\mathbf{n})}^{2}=\mathbf{n} \cdot \mathbf{B}^{-1} \mathbf{n}=n_{i} B_{i j}^{-1} n_{j} .
$$

If the element that is along $\mathbf{N}$ before deformation is along $\mathbf{n}$ after deformation, then [8]

$$
\lambda_{(\mathbf{N})} \lambda_{(\mathbf{n})}=1 .
$$

The shear $\gamma_{(\mathbf{M}, \mathbf{N})}$ of a pair of infinitesimal material line elements along the unit vectors $\mathbf{M}$ and $\mathbf{N}$ which subtend an angle $\Theta$ at $\mathbf{X}$ is given by [7]

$$
\cos \left(\Theta+\gamma_{(\mathbf{M}, \mathbf{N})}\right)=\mathbf{M} \cdot \mathbf{C N} /\left\{\lambda_{(\mathbf{M})} \lambda_{(\mathbf{N})}\right\} .
$$


Similarly, two infinitesimal material line elements along the unit vectors $\mathbf{m}$ and $\mathbf{n}$ which subtend the angle $\theta$ at $\mathbf{x}$ have been subjected to the shear $\gamma_{(\mathbf{m}, \mathbf{n})}$, given by

$$
\cos \left(\theta-\gamma_{(\mathbf{m}, \mathbf{n})}\right)=\mathbf{m} \cdot \mathbf{B}^{-1} \mathbf{n} /\left\{\lambda_{(\mathbf{m})} \lambda_{(\mathbf{n})}\right\} .
$$

If the elements along $\mathbf{M}, \mathbf{N}$ before deformation are along $\mathbf{m}, \mathbf{n}$ after deformation, then $\gamma_{(\mathbf{m}, \mathbf{n})}=$ $\gamma(\mathbf{M}, \mathbf{N}) \cdot$

Turning now to areal shear [9], let $\mathbf{P}$ and $\mathbf{Q}$ be unit normals to infinitesimal material planar elements at $\mathbf{X}$ subtending the angle $\Psi$ before deformation. If, after deformation, these material planar elements at $\mathbf{x}$ have unit normals $\mathbf{p}$ and $\mathbf{q}$, these subtend the angle $\psi=\Psi+\alpha(\mathbf{P}, \mathbf{Q})$, given by $[9]$

$$
\cos \left(\Psi+\alpha_{(\mathbf{P}, \mathbf{Q})}\right)=\mathbf{p} \cdot \mathbf{q}=\mathbf{P} \cdot \mathbf{C}^{-1} \mathbf{Q} /\left\{\mu_{(\mathbf{P})} \mu_{(\mathbf{Q})}\right\}, \quad \mu^{2}(\mathbf{P})=\mathbf{P} \cdot \mathbf{C}^{-1} \mathbf{P} .
$$

Also, the dual relation is

$$
\cos \left(\psi-\alpha_{(\mathbf{p}, \mathbf{q})}\right)=\mathbf{P} \cdot \mathbf{Q}=\mathbf{p} \cdot \mathbf{B q} /\left\{\mu_{(\mathbf{p})} \mu_{(\mathbf{q})}\right\}, \quad \mu^{2}(\mathbf{p})=\mathbf{p} \cdot \mathbf{B} \mathbf{p} .
$$

The "areal stretch" $\mu_{(\mathbf{P})}$ and the "areal resile" $\mu_{(\mathbf{p})}$ are related by $\mu_{(\mathbf{P})} \mu_{(\mathbf{p})}=1$, and the angle $\alpha_{(\mathbf{p}, \mathbf{q})}=\alpha_{(\mathbf{P}, \mathbf{Q})}$ is the "areal shear".

In the context of infinitesimal strain theory, the change in length per unit initial length of a material line element along $\mathbf{N}$ in the undeformed state is $e_{(\mathbf{N})}$ given by [10]

$$
e_{(\mathbf{N})}=\mathbf{N} \cdot \mathbf{e N}=N_{A} e_{A B} N_{B}
$$

whilst the shear $\gamma_{(\mathbf{M}, \mathbf{N})}$ is given by [11]

$$
\gamma_{(\mathbf{M}, \mathbf{N})}=\sin \Theta\left\{e_{(\mathbf{A})}-e_{(\mathbf{G})}\right\}
$$

where the orthogonal unit vectors $\mathbf{A}, \mathbf{G}$, coplanar with $\mathbf{M}$ and $\mathbf{N}$, are the bisectors of the angle $\Theta$ between $\mathbf{M}$ and $\mathbf{N}$, given by

$$
2 \sin (\Theta / 2) \mathbf{A}=\mathbf{M}-\mathbf{N}, \quad 2 \cos (\Theta / 2) \mathbf{G}=\mathbf{M}+\mathbf{N}
$$

Finally, we note that in the context of fluid motion, the stretching $d_{(\mathbf{n})}$ of an infinitesimal material line element instantaneously along the unit vector $\mathbf{n}$ at $\mathbf{x}$ at time $t$ is given by [7]

$$
d_{(\mathbf{n})}=\mathbf{n} \cdot \mathbf{d n}=n_{i} d_{i j} n_{j}
$$

whilst the shearing $\dot{\theta}_{(\mathbf{m}, \mathbf{n})}$, of a pair of material line elements instantaneously along the unit vectors $\mathbf{m}$ and $\mathbf{n}$, and subtending the angle $\theta$ at $\mathbf{x}$ at time $t$, is given by [11]

$$
\dot{\theta}_{(\mathbf{m}, \mathbf{n})}=\sin \theta\left\{d_{(\mathbf{a})}-d_{(\mathbf{g})}\right\},
$$

with $\mathbf{a}$ and $\mathbf{g}$ given by

$$
2 \sin (\theta / 2) \mathbf{a}=\mathbf{m}-\mathbf{n}, \quad 2 \cos (\theta / 2) \mathbf{g}=\mathbf{m}+\mathbf{n} .
$$




\section{Infinitesimal strain}

We first consider an isotropic compressible elastic material with constitutive equation

$$
t_{i j}=2 \mu e_{i j}+\lambda e_{k k} \delta_{i j},
$$

where $\lambda, \mu$ are constants. If the material is maintained in a state of simple shear or simple torsion, for each of which $e_{k k}=0$, so that there is no change in volume in the deformation, then, as Ting has pointed out [4], there exists an orthonormal triad $\mathbf{m}, \mathbf{n}, \mathbf{p}$ such that

$$
e_{(\mathbf{m})}=e_{(\mathbf{n})}=e_{(\mathbf{p})}=0 .
$$

Thus there is no change in length of material line elements initially along $\mathbf{m}, \mathbf{n}, \mathbf{p}$. It follows immediately that

$$
\mathbf{p} \cdot(\mathbf{e m} \times \mathbf{e n})+(\mathbf{m} \cdot \mathbf{e n})^{2}=0, \mathbf{m} \cdot(\mathbf{e n} \times \mathbf{e p})+(\mathbf{n} \cdot \mathbf{e p})^{2}=0, \mathbf{n} \cdot(\mathbf{e p} \times \mathbf{e m})+(\mathbf{p} \cdot \mathbf{e m})^{2}=0 .
$$

Also, using (3.1) and (3.2),

$$
\mathbf{m} \cdot \mathbf{t m}=\mathbf{n} \cdot \mathbf{t n}=\mathbf{p} \cdot \mathbf{t p}=0
$$

so that the material is in a state of pure shear. Of course from (3.1), we have $t_{k k}=0$, using $e_{k k}=0$. Similar to (3.3), we also have

$$
\mathbf{p} \cdot(\mathbf{t m} \times \mathbf{t n})+(\mathbf{m} \cdot \mathbf{t n})^{2}=0, \mathbf{m} \cdot(\mathbf{t n} \times \mathbf{t p})+(\mathbf{n} \cdot \mathbf{t} \mathbf{p})^{2}=0, \mathbf{n} \cdot(\mathbf{t} \mathbf{p} \times \mathbf{t m})+(\mathbf{p} \cdot \mathbf{t m})^{2}=0 .
$$

For an isotropic incompressible elastic material,

$$
t_{i j}=2 \mu e_{i j}+p \delta_{i j}, \quad e_{k k}=0,
$$

where $p$ is a scalar to be determined from the field equations and boundary/initial conditions. Because $e_{k k}=0$, it follows that there exists an orthonormal triad $\mathbf{m}, \mathbf{n}, \mathbf{p}$ (say) such that (3.2) holds. However, in the present case,

$$
\mathbf{m} \cdot \mathbf{t m}=\mathbf{n} \cdot \mathbf{t n}=\mathbf{p} \cdot \mathbf{t p}=p,
$$

which need not be zero, so that the conditions for pure shear do not hold. Even so, the universal relations

$$
\mathbf{m} \cdot \mathbf{t m}-\mathbf{n} \cdot \mathbf{t n}=0, \quad \mathbf{n} \cdot \mathbf{t n}-\mathbf{p} \cdot \mathbf{t p}=0
$$

are valid.

Suppose now that $e_{k k}=0$ for a particular deformation or is a constraint on the deformation. In either case we have an orthonormal triad $\mathbf{m}, \mathbf{n}, \mathbf{p}$ for which (3.2) hold. If the unit vectors $\mathbf{r}$, $\mathbf{s}$ are given by

$$
\mathbf{r}=\cos (\phi / 2) \mathbf{m}-\sin (\phi / 2) \mathbf{n}, \quad \mathbf{s}=\cos (\phi / 2) \mathbf{m}+\sin (\phi / 2) \mathbf{n},
$$

where $\phi$ is arbitrary, then $\gamma_{(\mathbf{r}, \mathbf{s})}$, the shear of the pair of infinitesimal material line elements along $\mathbf{r}$ and $\mathbf{s}$ is

$$
\gamma_{(\mathbf{r}, \mathbf{s})}=\sin \phi\left\{e_{(\mathbf{m})}-e_{(\mathbf{n})}\right\}=0,
$$

using (3.2). Thus the shear is zero for any pair $(\mathbf{r}, \mathbf{s})$ of material line elements given by (3.9) in which $\phi$ is arbitrary, hence any pair $(\mathbf{r}, \mathbf{s})$ such that $\mathbf{m}$ and $\mathbf{n}$ are along the bisectors of the angle $\phi$ subtended by this pair. 
Similarly, there is an infinity of unsheared pairs along $\left(\mathbf{r}^{\prime}, \mathbf{s}^{\prime}\right)$ in the plane of $(\mathbf{n}, \mathbf{p})$ given by

$$
\mathbf{r}^{\prime}=\cos \left(\phi^{\prime} / 2\right) \mathbf{n}-\sin \left(\phi^{\prime} / 2\right) \mathbf{p}, \quad \mathbf{s}^{\prime}=\cos \left(\phi^{\prime} / 2\right) \mathbf{n}+\sin \left(\phi^{\prime} / 2\right) \mathbf{p},
$$

where $\phi^{\prime}$ is arbitrary, and along $\left(\mathbf{r}^{\prime \prime}, \mathbf{s}^{\prime \prime}\right)$ in the plane of $(\mathbf{p}, \mathbf{m})$ given by

$$
\mathbf{r}^{\prime \prime}=\cos \left(\phi^{\prime \prime} / 2\right) \mathbf{p}-\sin \left(\phi^{\prime \prime} / 2\right) \mathbf{m}, \quad \mathbf{s}^{\prime \prime}=\cos \left(\phi^{\prime \prime} / 2\right) \mathbf{p}+\sin \left(\phi^{\prime \prime} / 2\right) \mathbf{m},
$$

where $\phi^{\prime \prime}$ is arbitrary.

From (3.1), in the case of a compressible material, we note for the stress that we have

$$
\mathbf{r} \cdot \mathbf{t s}=\mathbf{r}^{\prime} \cdot \mathbf{t s}^{\prime}=\mathbf{r}^{\prime \prime} \cdot \mathbf{t s}^{\prime \prime}=0
$$

In the case of an incompressible material, from (3.6), we have

$$
\mathbf{r} \cdot \mathbf{t s}=p \mathbf{r} \cdot \mathbf{s}, \quad \mathbf{r}^{\prime} \cdot \mathbf{t s} \mathbf{s}^{\prime}=p \mathbf{r}^{\prime} \cdot \mathbf{s}^{\prime}, \quad \mathbf{r}^{\prime \prime} \cdot \mathbf{t s} \mathbf{s}^{\prime \prime}=p \mathbf{r}^{\prime \prime} \cdot \mathbf{s}^{\prime \prime},
$$

so that

$$
\frac{\mathbf{r} \cdot \mathbf{t s}}{\mathbf{r} \cdot \mathbf{s}}=\frac{\mathbf{r}^{\prime} \cdot \mathbf{t s}^{\prime}}{\mathbf{r}^{\prime} \cdot \mathbf{s}^{\prime}}=\frac{\mathbf{r}^{\prime \prime} \cdot \mathbf{t s}^{\prime \prime}}{\mathbf{r}^{\prime \prime} \cdot \mathbf{s}^{\prime \prime}}
$$

Of course, when $\phi=\pi / 2\left(\phi^{\prime}=\pi / 2, \phi^{\prime \prime}=\pi / 2\right)$, then $\mathbf{r} \cdot \mathbf{t s}=0\left(\mathbf{r}^{\prime} \cdot \mathbf{t s}^{\prime}=0, \mathbf{r}^{\prime \prime} \cdot \mathbf{t s}^{\prime \prime}=0\right)$.

\section{$4 \quad$ Finite strain}

Turning now to the theory of finite strain we consider two models of homogeneous isotropic elastic materials, the Bell material [12] and the Ericksen material [13]. These are models of internally constrained materials. It is observed that the internal constraints may be written in the form $\operatorname{tr} \boldsymbol{\Delta}=0$, where $\boldsymbol{\Delta}$ is a symmetric second order tensor, which means that there exists an orthonormal triad such that the diagonal components of $\boldsymbol{\Delta}$ in this triad are all zero. We explore the consequences of this fact for the two constraints.

The Bell model is such that the material is assumed to be subject to the internal constraint

$$
\operatorname{tr} \mathbf{V}=3
$$

where $\mathbf{V}=\mathbf{B}^{1 / 2}$, so that the sum of the principal stretches always adds up to three in any deformation. The constitutive equation is

$$
\mathbf{t}=-p \mathbf{V}+\alpha \mathbf{1}+\beta \mathbf{B},
$$

where $\alpha, \beta$ are functions of the invariants $\left(\operatorname{tr} \mathbf{B}, \operatorname{tr} \mathbf{B}^{2}\right)$ and $p$ is to be determined from the field equations and boundary/initial conditions.

We note that (4.1) may be written

$$
\operatorname{tr}(\mathbf{V}-\mathbf{1})=0, \quad \text { or, equivalently, } \operatorname{tr}(\mathbf{U}-\mathbf{1})=0,
$$

where $\mathbf{U}=\mathbf{C}^{1 / 2}$. From (4.3) it follows that there exists an orthonormal triad $\mathbf{m}, \mathbf{n}, \mathbf{p}$ (say) such that

$$
\mathbf{m} \cdot \mathbf{V m}=\mathbf{n} \cdot \mathbf{V n}=\mathbf{p} \cdot \mathbf{V} \mathbf{p}=1
$$


It then follows from (4.2) that

$$
\frac{\mathbf{n} \cdot \mathbf{t n}-\mathbf{m} \cdot \mathbf{t m}}{\mathbf{m} \cdot \mathrm{tm}-\mathrm{p} \cdot \mathrm{t} \mathbf{p}}=\frac{\mathbf{n} \cdot \mathrm{Bn}-\mathbf{m} \cdot \mathrm{Bm}}{\mathrm{m} \cdot \mathrm{Bm}-\mathrm{p} \cdot \mathrm{Bp}}
$$

a universal relation [14] valid for any deformation of the material. Also, using (4.4), we have

$$
\mathbf{u} \cdot \mathbf{V w}=0
$$

where $\mathbf{u}, \mathbf{w}$, orthogonal unit vectors, are given by

$$
\sqrt{2} \mathbf{u}=\mathbf{m}+\mathbf{n}, \quad \sqrt{2} \mathbf{w}=\mathbf{m}-\mathbf{n} .
$$

Similarly, $\mathbf{u}^{\prime} \cdot \mathbf{V} \mathbf{w}^{\prime}=0$ and $\mathbf{u}^{\prime \prime} \cdot \mathbf{V} \mathbf{w}^{\prime \prime}=0$, where $\mathbf{u}^{\prime}, \mathbf{v}^{\prime}$ are unit vectors along the bisectors of $(\mathbf{n}, \mathbf{p})$ at $\mathbf{x}$, and $\mathbf{u}^{\prime \prime}, \mathbf{v}^{\prime \prime}$ are unit vectors along the bisectors of $(\mathbf{p}, \mathbf{m})$ at $\mathbf{x}$. From the constitutive equation (4.2), we then note that

$$
\mathbf{u} \cdot \mathbf{t w}=\beta \mathbf{u} \cdot \mathbf{B w}, \quad \mathbf{u}^{\prime} \cdot \mathbf{t} \mathbf{w}^{\prime}=\beta \mathbf{u}^{\prime} \cdot \mathbf{B} \mathbf{w}^{\prime}, \quad \mathbf{u}^{\prime \prime} \cdot \mathbf{t} \mathbf{w}^{\prime \prime}=\beta \mathbf{u}^{\prime \prime} \cdot \mathbf{B} \mathbf{w}^{\prime \prime}
$$

so that we have the universal relations

$$
\frac{\mathrm{u} \cdot \mathrm{tw}}{\mathrm{u} \cdot \mathrm{Bw}}=\frac{\mathbf{u}^{\prime} \cdot \mathbf{t w}^{\prime}}{\mathbf{u}^{\prime} \cdot \mathrm{Bw}^{\prime}}=\frac{\mathrm{u}^{\prime \prime} \cdot \mathbf{t w}}{\mathbf{u}^{\prime \prime} \cdot \mathrm{Bw}^{\prime \prime}} .
$$

Because there is an infinity of orthonormal triads for which (4.4) holds, there is a corresponding infinity of unit vectors $\mathbf{u}, \mathbf{v}, \mathbf{u}^{\prime}, \mathbf{v}^{\prime}, \mathbf{u}^{\prime \prime}, \mathbf{v}^{\prime \prime}$ for which these relations are valid.

In the Ericksen model, the material is assumed to be subject to the internal constraint

$$
\operatorname{tr} \mathbf{C}=3, \quad \text { or, equivalently, } \operatorname{tr} \mathbf{B}=3 .
$$

so that the sum of squares of the principal stretches always adds up to three. The constitutive equation is

$$
\mathbf{t}=-p \mathbf{B}+\gamma \mathbf{1}+\delta \mathbf{B}^{-1}
$$

where $\gamma, \delta$ are functions of the invariants $\left(\operatorname{tr} \mathbf{B}^{2}, \operatorname{tr} \mathbf{B}^{3}\right)$ and $p$ is to be determined from the field equations and boundary/initial conditions.

We note that (4.10) may be written

$$
\operatorname{tr}(\mathbf{C}-\mathbf{1})=0, \quad \text { or, equivalently, } \operatorname{tr}(\mathbf{B}-\mathbf{1})=0 .
$$

Hence, from $(4.12)_{1}$, there exists an orthonormal triad $\mathbf{M}, \mathbf{N}, \mathbf{P}$ (say) at $\mathbf{X}$ such that

$$
\mathbf{M} \cdot \mathbf{C M}=\mathbf{N} \cdot \mathbf{C N}=\mathbf{P} \cdot \mathbf{C P}=1
$$

or, equivalently,

$$
\lambda_{(\mathbf{M})}^{2}=\lambda_{(\mathbf{N})}^{2}=\lambda_{(\mathbf{P})}^{2}=1,
$$

so that the infinitesimal material line elements initially along $\mathbf{M}, \mathbf{N}, \mathbf{P}$ (say) are unstretched in the deformation. Also, using (4.13) we have

$$
\mathbf{R} \cdot \mathbf{C S}=0
$$


where $\mathbf{R}, \mathbf{S}$, orthogonal unit vectors along the bisectors, internal and external, of the right angle subtended by $\mathbf{M}$ and $\mathbf{N}$, are given by

$$
\sqrt{2} \mathbf{R}=\mathbf{M}+\mathbf{N}, \quad \sqrt{2} \mathbf{S}=\mathbf{M}-\mathbf{N} .
$$

Thus, using (2.4),

$$
\gamma_{(\mathbf{R}, \mathbf{S})}=0
$$

the pair of orthogonal material line elements initially along $\mathbf{R}, \mathbf{S}$, the bisectors of the angle between $\mathbf{M}$ and $\mathbf{N}$ at $\mathbf{X}$, is unsheared.

Similarly, the pairs of orthogonal material line elements initially along the bisectors of the angle between $\mathbf{N}$ and $\mathbf{P}$, and between $\mathbf{P}$ and $\mathbf{M}$, are unsheared.

From $(4.12)_{2}$, it follows that there exists an orthonormal triad $\mathbf{f}, \mathbf{g}, \mathbf{h}$ (say) at $\mathbf{x}$ such that

$$
\mathbf{f} \cdot \mathbf{B f}=\mathbf{g} \cdot \mathbf{B g}=\mathbf{h} \cdot \mathbf{B h}=1
$$

It then follows from the constitutive equation (4.11) that we have the universal relation

$$
\frac{\mathbf{f} \cdot \mathbf{t f}-\mathrm{g} \cdot \mathrm{tg}}{\mathrm{g} \cdot \mathrm{tg}-\mathrm{h} \cdot \mathrm{th}}=\frac{\mathbf{f} \cdot \mathrm{B}^{-1} \mathbf{f}-\mathrm{g} \cdot \mathrm{B}^{-1} \mathrm{~g}}{\mathrm{~g} \cdot \mathrm{B}^{-1} \mathrm{~g}-\mathrm{h} \cdot \mathrm{B}^{-1} \mathbf{h}}
$$

valid for any deformation of the material. Also, using (4.18), we have

$$
\mathbf{k} \cdot \mathbf{B l}=0
$$

where $\mathbf{k}, \mathbf{l}$, orthogonal unit vectors along the bisectors, internal and external, of the right angle subtended by $\mathbf{f}$ and $\mathbf{g}$, are given by

$$
\sqrt{2} \mathbf{k}=\mathbf{f}+\mathbf{g}, \quad \sqrt{2} \mathbf{l}=\mathbf{f}-\mathbf{g} .
$$

Thus, using (2.7),

$$
\alpha_{(\mathbf{k}, \mathbf{l})}=0
$$

the pair of material planar elements with unit normals $\mathbf{k}, \mathbf{l}$ at $\mathbf{x}$ has suffered no planar shear. Similarly, $\mathbf{k}^{\prime} \cdot \mathbf{B l}^{\prime}=0$ and $\mathbf{k}^{\prime \prime} \cdot \mathbf{B l}^{\prime \prime}=0$, where $\mathbf{k}^{\prime}, \mathbf{l}^{\prime}$ are unit vectors along the bisectors of $(\mathbf{g}, \mathbf{h})$ at $\mathbf{x}$, and $\mathbf{k}^{\prime \prime}, \mathbf{l}^{\prime \prime}$ are unit vectors along the bisectors of $(\mathbf{h}, \mathbf{f})$ at $\mathbf{x}$. From the constitutive equation (4.11), we then note that

$$
\mathbf{k} \cdot \mathbf{t l}=\beta \mathbf{k} \cdot \mathbf{B}^{-1} \mathbf{l}, \quad \mathbf{k}^{\prime} \cdot \mathbf{t l}^{\prime}=\beta \mathbf{k}^{\prime} \cdot \mathbf{B}^{-1} \mathbf{l}^{\prime}, \quad \mathbf{k}^{\prime \prime} \cdot \mathbf{t} \mathbf{l}^{\prime \prime}=\beta \mathbf{k}^{\prime \prime} \cdot \mathbf{B}^{-1} \mathbf{l}^{\prime \prime},
$$

so that we have the universal relations

$$
\frac{\mathbf{k} \cdot \mathbf{t l}}{\mathrm{k} \cdot \mathbf{B}^{-1} \mathbf{l}}=\frac{\mathbf{k}^{\prime} \cdot \mathbf{t l}^{\prime}}{\mathbf{k}^{\prime} \cdot \mathbf{B}^{-1} \mathbf{l}^{\prime}}=\frac{\mathbf{k}^{\prime \prime} \cdot \mathbf{t l}^{\prime \prime}}{\mathbf{k}^{\prime \prime} \cdot \mathbf{B}^{-1} \mathbf{l}^{\prime \prime}}
$$

Because there is an infinity of orthonormal triads for which (4.18) holds, there is a corresponding infinity of unit vectors $\mathbf{k}, \mathbf{l}, \mathbf{k}^{\prime}, \mathbf{l}^{\prime}, \mathbf{k}^{\prime \prime}, \mathbf{l}^{\prime \prime}$ for which these relations are valid. 


\section{Fluid motion}

If a fluid is incompressible or the motion of a fluid is isochoric, then in either case,

$$
\operatorname{tr} \mathbf{d}=0
$$

where $\mathbf{d}$ is the rate of strain tensor. It follows that there exists an orthonormal triad $\mathbf{m}, \mathbf{n}, \mathbf{p}$ (say) such that

$$
\mathbf{m} \cdot \mathbf{d} \mathbf{m}=\mathbf{n} \cdot \mathbf{d n}=\mathbf{p} \cdot \mathbf{d p}=0,
$$

that is, the stretchings along $\mathbf{m}, \mathbf{n}$ and $\mathbf{p}$ are all zero. For the unit vectors $\mathbf{r}$ and $\mathbf{s}$ subtending the arbitrary angle $\phi$ and given by (3.9), the instantaneous shearing $\dot{\theta}_{(\mathbf{m}, \mathbf{n})}$ of the pair of infinitesimal material line elements along $(\mathbf{r}, \mathbf{s})$ at $\mathbf{X}$ at time $t$ is given by

$$
\dot{\theta}_{(\mathbf{r}, \mathbf{s})}=\sin \phi\left\{d_{(\mathbf{m})}-d_{(\mathbf{n})}\right\}=0 .
$$

Thus, the shearing $\dot{\theta}_{(\mathbf{r}, \mathbf{s})}$ is zero for all pairs of material line elements at $\mathbf{x}$, at time $t$, instantaneously symmetrically disposed with respect to $\mathbf{m}$ and $\mathbf{n}$. Similar statements are valid for all pairs symmetrically disposed with respect to $\mathbf{n}$ and $\mathbf{p}(\mathbf{p}$ and $\mathbf{m}$ ) in the plane of $\mathbf{n}$ and $\mathbf{p}$ ( $\mathbf{p}$ and $\mathbf{m})$, instantaneously at $\mathbf{x}$ at time $t$.

Suppose the constitutive equation for the fluid is

$$
t_{i j}=-p \delta_{i j}+2 \mu d_{i j}, \quad d_{k k}=0,
$$

where $\mu$ is a constant and $p$ is to be determined from the field equations and boundary/initial conditions. Then,

$$
\mathbf{m} \cdot \mathbf{t m}=\mathbf{n} \cdot \mathbf{t n}=\mathbf{p} \cdot \mathbf{t p}=p,
$$

so that, in particular, the normal components of traction along $\mathbf{m}, \mathbf{n}, \mathbf{p}$ are related through

$$
\mathbf{m} \cdot \mathbf{t m}-\mathbf{n} \cdot \mathbf{t n}=0, \quad \mathbf{n} \cdot \mathbf{t n}-\mathbf{p} \cdot \mathbf{t p}=0,
$$

valid for every motion of the fluid.

\section{References}

[1] M. Gurtin, The linear theory of elasticity. Flugge's Handbuch der Physik, VIa/2. Springer Verlag, Berlin-Heidelberg-New York (1972).

[2] P. Belik and R. Fosdick, The state of pure shear. J. of Elasticity 52, 91-98, (1998).

[3] Ph. Boulanger and M. Hayes, On pure shear. J. of Elasticity 77, 83-89, (2004).

[4] T. C. T. Ting, Further study on pure shear. J. of Elasticity 83, 95-104, (2006).

[5] A. Norris, Pure shear axes and elastic strain energy. Q. Jl. Mech. appl Math., to appear.

[6] A. J. McConnell, Applications of Tensor Analysis, Dover Publications (1957).

[7] W. Thomson (Lord Kelvin) and P. G. Tait, Treatise on Natural Philosophy. Part I, Cambridge University Press, Cambridge (1923). 
[8] Ph. Boulanger and M. Hayes, Extended polar decompositions for plane strain. J. of Elasticity 83, 29-64, (2006).

[9] Ph. Boulanger and M. Hayes, On finite shear. Arch. Rational Mech. Anal. 100, 265-273, (1988).

[10] A. E. H. Love, The Mathematical Theory of Elasticity, 4th edition (1927), Dover Publications (2003).

[11] Ph. Boulanger and M. Hayes, Shear, shear stress and shearing. J. Mech. Phys. solids 40, 1449-1457, (1992).

[12] M. F. Beatty and M. Hayes, Deformations of an elastic, internally constrained material. Part 1: Homogeneous deformations. J. of Elasticity 29, 1-84, (1992).

[13] J. L. Ericksen, Constitutive Theory for Some Constrained Elastic Crystals. Preprint No.132, Institute for Mathematics and its Applications, University of Minnesota, Minneapolis, (1985).

[14] R. S. Rivlin, Some applications of elasticity theory to rubber engineering. Proc. 2nd Tech. Conf. (London, June 23-25, 1948), Cambridge: Heffer (1948). 